\title{
EVALUATION OF OUTCOME OF VARIOUS TREATMENT METHODS FOR ENDOGENOUS ENDOPHTHALMITIS
}

\author{
TULSI KESWANI, VIJAY AHUJA*, MANISH CHANGULANI**
}

\section{ABSTRACT}

\begin{abstract}
AIM: To evaluate the role of different treatment methods (intravitreal antibiotics and vitrectomy) for endogenous endophthalmitis and assess the outcome. MATERIALS AND METHODS: This is a retrospective observational case study. Sixteen cases of endogenous endophthalmitis noted in 14 patients between October 2000 and April 2004 were reviewed. Two patients had bilateral disease. Patients were followed up for a mean of 3 months (range, 1-12 months). RESULTS: Vitreous and aqueous cultures were positive in $8(66 \%)$ cases and $3(75 \%)$ cases respectively. Fungal isolates occurred in $4(33 \%)$ cases, out of which 3 were due to candida, gram-positive isolates occurred in $2(16 \%)$ and gram-negative isolates in $2(16 \%)$ of the cases. Initial treatment included vitreous tap and injection of intravitreal medication in $12(75 \%)$ cases and pars plana vitrectomy with injection of intravitreal medication in $3(19 \%)$ cases. Final visual outcomes were obtainable for 13 cases (1 patient died and 1 patient was lost to followup and 1 eye was eviscerated). Five (43\%) of these cases achieved visual acuity of $6 /$ 60 or better and 8 (56\%) eyes achieved a visual acuity less than 6/60. Risk factors mainly associated with the disease were uncontrolled diabetes mellitus, chronic renal failure, renal transplant, abdominal surgeries and septicemia. CONCLUSION: Our experience suggests that endogenous endophthalmitis is generally associated with poor visual outcome. Prompt treatment with intravitreal antibiotics and vitrectomy can result in improvement in ocular signs and visual acuity in majority of the patients. The patients with culture-positive endogenous endophthalmitis are more likely to have fungal isolates with a predominance of candida. Fungal endopthalmitis has a poor visual outcome as compared to bacterial endophthalmitis.
\end{abstract}

Key words: Endogenous, endophthalmitis, intravitreal antibiotics, vitrectomy

Endogenous endophthalmitis is a potentially blinding intraocular infection resulting from the hematogenous spread of organisms from a distant source of infection (e.g.,

National Institute of Ophthalmology, Pune, *Bombay Hospital, Indore, ${ }^{\star *}$ King George Medical College, Lucknow, India

\section{Correspondence}

Dr. Tulsi Keswani, 31, Fern House, Amberley Drive, Manchester, United Kingdom. E-mail: tulsikes@yahoo.com endocarditis). It is relatively rare, accounting for $2-8 \%$ of all cases of endophthalmitis and is associated with immunocompromised state, debilitating diseases and invasive procedures..$^{[1]}$ Due to the rapid advance of medical technology, a longer lifespan of patients with chronic disease and a rising prevalence of long-term intravenous access, the disease may become more common in 
clinical practice.

Many etiological organisms (gram-positive, gram-negative and fungal) have been reported to cause endogenous endophthalmitis. ${ }^{[2,3]} \mathrm{A}$ high clinical suspicion is needed for early diagnosis and treatment. A classification system introduced by Greenwald ${ }^{[4]}$ takes into consideration the affected areas of the globe and the associated visual prognosis. Focal endophthalmitis responds well to intravenous antibiotics and generally results in minimal sequelae. Posterior diffuse endophthalmitis and panophthalmitis are associated with a much poorer prognosis; often these conditions lead to blindness, atrophy of the globe or enucleation. Early intravenous antibiotic therapy remains the cornerstone of treatment. ${ }^{[4]}$ The role of intravitreal antibiotics and vitrectomy is evolving and they may become more widely accepted as therapeutic modalities. It is important that a general practitioner is aware of endogenous endophthalmitis because early diagnosis and prompt aggressive treatment are imperative if vision loss is to be avoided.

$$
\text { (2) }
$$

The objective of this study was to evaluate the role of intravitreal antibiotics and vitrectomy in improving final visual outcome in patients with endogenous endophthalmitis.

\section{MATERIALS AND METHODS}

This is a retrospective observational case study conducted at a hospital in Visakhapatnam, India. Fourteen patients of endogenous endophthalmitis seen between October 2000 and April 2004 were reviewed. Patients were considered to have endogenous endophthalmitis if at least one of the following three criteria were met:

a. Growth present on more than one media from vitreous or aqueous cultures

b. A positive blood culture result

c. Posterior and/or anterior ocular inflammation

All patients underwent detailed clinical ocular examinations (visual acuity by Snellen chart, slit lamp biomicroscopy and indirect ophthalmoscopy). All patients were also seen by the concerned physician to find out the source of infection. All diagnostic tests, chest X-ray, CT scan of orbit, cardiac ultrasound and echocardiography were done, which were helpful to find out the focus of infection. The culture and sensitivity of non-ocular sites like blood and urine were done. Blood culture was done thrice in all cases. ${ }^{[5]}$ Aqueous and vitreous cultures were also done whenever possible by an ophthalmic surgeon; sterile needles were used to aspirate material. Because of the small amounts of material involved, inoculation of plates and preparation of slides were done at the patient's side.

The initial vitreous aspirates were sent for smear, culture (bacterial and fungus) and antibacterial sensitivity. These samples were subjected to Gram stain, Periodic Acid Schiff stain and calcofluor stains for direct microscopy under light and fluorescent microscope and cultured on $5 \%$ blood and chocolate agar (any organism), anaerobic thioglycollate broth and Sabourauds agar media (fungi) ${ }^{[6]}$ A standard clinical pro forma was used for all cases for analytic study, which included age, sex, clinical findings, ancillary test and anatomical type of endophthalmitis.

The results were analyzed using descriptive statistics and frequency analysis.

\section{RESULTS}

Fourteen patients of endogenous endophthalmitis were analyzed in this study. Eight $(57 \%)$ of the 14 patients were males and $6(43 \%)$ were females [Table 1]. Average age at time of presentation was 61 years with a range of 20 to 75 years. Five patients belonged to the age group of 60-70 years, 5 to $41-60$ years, 3 to $21-40$ years and only 1 patient to $0-20$ years. unilateral involvement (5 right eyes and 7 left eyes were in the current series) and 2 patients had bilateral involvement. All patients had history of other systemic illnesses. Of these 14 patients, 6 had diabetes, 3 had CRF, 1 had chest infection, drugs, 1 had endocarditis, 1 had hepatitis, 1 had abdominal surgery, 1 had head injury and
Out of the 14 patients, 12 patients had 2 had UTI, 2 were on immunosuppressive

1 had meningitis.

Patients were referred to us or came directly at varying periods - between 1 and 14 days after the onset of symptoms. Out of 14 patients, 9 were already admitted in acute medical care and 5 came directly to ophthalmology outpatient department. Examination findings were recorded [Table 2]. Eight $(50 \%)$ out of 16 patients presented with focal posterior endophthalmitis, 4 (25\%) with diffuse posterior endophthalmitis and 4 (25\%) with combined (anterior and posterior) diffuse endophthalmitis.

Four $(25 \%)$ patients had hypopyon in addition to vitreous exudates.

Out of 14 patients, only 1 (7\%) patient had positive blood culture, $2(14 \%)$ had positive urine culture, $1(7 \%)$ had positive wound culture, $1(7 \%)$ had CSF culture positive and one $(7 \%)$ patient had positive culture on catheter tip [Table 3].

Out of 16 eyes, $12(75 \%)$ eyes were treated with intravitreal (vancomycin-amikacin) and

\section{Table 1: Clinical details Risk factors of patients with endogenous endophthalmitis}

\begin{tabular}{|c|c|c|c|c|}
\hline Patient no. & $\begin{array}{c}\text { Age } \\
(y r s) / s e x\end{array}$ & Eye & $\begin{array}{l}\text { Duration } \\
\text { (days) }\end{array}$ & Risk factors \\
\hline 1 & $48 / \mathrm{M}$ & $\mathrm{R}$ & 5 & Uncontrolled DM \\
\hline 2 & $55 / M$ & L & 7 & DM, Renal transplantation \\
\hline 3 & $37 / \mathrm{F}$ & $\mathrm{R}$ & 2 & Multiple abdominal surgeries \\
\hline 4 & $47 / F$ & $B / L$ & & Uncontrolled DM, ARF, UTI \\
\hline 5 & $62 / F$ & $\mathrm{~L}$ & No symptoms & Endocarditis \\
\hline 6 & $64 / F$ & $\mathrm{~L}$ & 2 & Uncontrolled DM, CRF, Septicaemia \\
\hline 7 & $12 / \mathrm{M}$ & $B / L$ & No symptoms & Meningitis \\
\hline 8 & $37 / \mathrm{F}$ & $\mathrm{L}$ & 8 & SLE on systemic steroids \\
\hline 9 & $64 / \mathrm{M}$ & L & 10 & DM, recurrent UTI \\
\hline 10 & 70/F & $\mathrm{R}$ & 5 & DM, Ulcer, CRF on haemodialysis \\
\hline 11 & $36 / \mathrm{M}$ & $\mathrm{L}$ & 5 & Chest infection \\
\hline 12 & 44/M & $\mathrm{R}$ & 10 & Alcohol hepatitis \\
\hline 13 & 48/M & L & 4 & Head injury, on ventilator \\
\hline 14 & $66 / \mathrm{M}$ & $\mathrm{R}$ & 7 & Old pulmonary tuberculosis \\
\hline
\end{tabular}

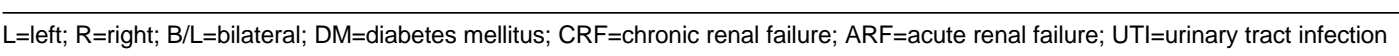


Table 2: Initial ocular examination at time of endophthalmitis diagnosis and initial management

\begin{tabular}{lccc}
\hline Patient no & Clinical signs & Type of treatment given & Endophthalmitis \\
\hline 1 & Hypopyon, retinal and vitreous exudates (arcade) & Diffuse-anterior and posterior & A, B, C \\
2 & Hypopyon and retinal exudates (arcade) & Diffuse-anterior and posterior & A, B, C \\
3 & Retinal exudate (arcade) & Focal-posterior & B, C \\
4 & Retinal exudate (arcade) & Diffuse-posterior & B (Both eyes), C \\
5 & Retinal exudate-roth spots & Focal-posterior & C \\
6 & Hypopyon,Vitritis, vitreous haemorrhage & Diffuse & B, C \\
7 & Retinal exudates (arcade) & Focal-posterior & C \\
8 & Retinal exudates (arcade) & Focal-posterior & B, C \\
9 & Retinal (arcade) and vitreal exudates & Diffuse-posterior & A, B, C \\
10 & Retinal (arcade) and vitreal exudates & Focal-posterior & B, C \\
11 & Retinal (arcade) exudate & Focal-posterior & B, C \\
12 & Hypopyon, Retinal and vitreous exudates & Diffuse-anterior and posterior & B, C \\
13 & Retinal exudate (arcade) & Focal-posterior & C (Both eyes) \\
14 & Retinal exudate (arcade) & Focal-posterior & B, C \\
\hline
\end{tabular}

$\mathrm{A}=$ Pars plana vitrectomy, $\mathrm{B}=$ Intravitreal antibiotics, $\mathrm{C}=$ Intravenous antibiotics

Table 3: Culture results of patients with endogenous endophthalmitis

\begin{tabular}{|c|c|c|c|c|}
\hline Patient no. & $\begin{array}{c}\text { Vitreous/aqueous } \\
\text { culture }\end{array}$ & $\begin{array}{l}\text { Blood /urine } \\
\text { culture }\end{array}$ & $\begin{array}{l}\text { Primary source } \\
\text { of Infection }\end{array}$ & $\begin{array}{c}\text { Supportive } \\
\text { test }\end{array}$ \\
\hline 1 & Candida & No growth & Not found & NS \\
\hline 2 & Candida & No growth & Not found & NS \\
\hline 3 & Staphylococcus & No growth & intestine & Neutrophilia aureus \\
\hline 4 & One eye-Gm+ & urine culture+ & catheter & Neutrophilia \\
\hline 5 & Not performed & No growth & Heart & Echocardiography \\
\hline 6 & Pseudomonas & No growth & Not found & NS \\
\hline 7 & Not performed & SF-pnemococcu & Chest? & NS \\
\hline 8 & Not performed & Blood culture+ & Not found & NS \\
\hline 9 & & Urine culture- & & Staph aureus \\
\hline & Aspergillus & & Not found & E. coli \\
\hline 10 & Candida? & No growth & Not found & NS \\
\hline 11 & Not performed & No growth & Chest infection & NS \\
\hline 12 & Klebsiella & No growth & Not found & NS \\
\hline 13 & Not performed & No growth & Not found & NS \\
\hline 14 & Not performed & No growth & Not found & NS \\
\hline
\end{tabular}

NS=not significant

intravenous antibiotics (vancomycin + ceftazidime) and 4 (25\%) patients were managed by intravenous antibiotics only (vancomycin-ceftazidime) [Table 2]. Out of these 12 patients, who were treated with intravitreal antibiotics, 8 (66\%) were culturepositive (intravitreal) and the rest 4 (33\%) were culture-negative.

Out of the 8 patients who were culture positive, 4 were positive for fungus ( 3 , candida; 1, aspergillus), 2 for gram-positive
(1, S. aureus; 1 , Streptococcus pneumoniae) and the remaining 2 for gram negative (1, Pseudomonas; 1, Klebsiella).

Anterior chamber tap was done for (4 eyes) hypopyon for anterior chamber cleaning as well as for therapeutic purposes and was sent for microbiology. Three cases were culturepositive (2 for fungus, 1 for gram-negative) and 1 was culture-negative.

Out of the 4 patients who were found to have fungal endophthalmitis, 3 patients underwent pars plana vitrectomy with injection of amphotericin B. One patient died due to septicemia.

All 14 patients (16 eyes) presented with visual acuity from hand movement to $6 / 36$ [Table 4]. Final visual outcomes were obtainable for 13 eyes ( 1 patient died due to septicemia, 1 patient was lost to follow-up and 1 eye was eviscerated). Overall, 5 (38\%) of 13 patients had final visual acuity of $6 / 60$ or better $(1-6 / 24,1-6 / 18,2-6 / 12$ and $1-6 /$ 9 ); the rest ( 8 eyes $-62 \%$ ) had visual acuity of less than $6 / 60$ (CF $1 \mathrm{~m}$ to $6 / 60$ ). It was observed that bacterial infection had better outcome as compared to fungal endophthalmitis. ${ }^{[7,8]}$

\section{DISCUSSION}

Metastatic endophthalmitis is a rare entity, which occurs at an average annual incidence of about 5 in 100,000 hospitalized patients. It can occur at any age, ranging from 1 week to 85 years. Bilateral involvement is seen in 14 to $25 \%$ of patients and reports have

\begin{tabular}{|c|c|c|}
\hline \multirow[t]{2}{*}{ Patient no. } & \multicolumn{2}{|c|}{ Visual acuity } \\
\hline & Pretreatment & Final \\
\hline 1 & $3 / 60$ & $5 / 60$ \\
\hline 2 & $\mathrm{CF}<1 \mathrm{~m}$ & $4 / 60$ \\
\hline 3 & CF $1 \mathrm{~m}$ & $6 / 60$ \\
\hline 4 & $6 / 36$ & $6 / 24$ \\
\hline 5 & CF $1 \mathrm{~m}$ & $6 / 12$ \\
\hline 6 & HM & Eviscerated \\
\hline 7 & $\mathrm{HM}$ & $6 / 9$ \\
\hline 8 & CF1m & $5 / 60$ \\
\hline 9 & $H M$ & $3 / 60$ \\
\hline 10 & $H M$ & Died \\
\hline 11 & $6 / 60$ & $6 / 18$ \\
\hline 12 & CF $1 \mathrm{~m}$ & Lost to followup \\
\hline 13 & HM & $5 / 60$ \\
\hline 14 & $6 / 36$ & $6 / 60$ due to cataract \\
\hline
\end{tabular}

$\mathrm{CF}=$ Counts fingers shown a higher incidence of involvement of right eye versus left eye ${ }^{[3]}$ in patients with unilateral disease. There is no sex preponderance. Once the diagnosis has been made or strongly considered, prompt consultation with an ophthalmologist is needed. Treatment depends on the underlying cause of endophthalmitis. Final visual outcome is heavily dependent on timely recognition and treatment.

Once the diagnosis of candidial endophthalmitis is made, admit the patient to the hospital, start intravenous or intravitrea amphotericin B and oral fluconazole.

\section{U.}

The prognosis is extremely variable because of the variety of organisms involved. The visual acuity at the time of diagnosis and the causative agent are most predictive of outcome.

The outcome of endogenous endophthalmitis is generally worse than exogenous endophthalmitis due to the profile of the organisms typically involved with this form (i.e., more virulent organisms, compromised host, delay in diagnosis). The prognosis appears to also be related to the patient's underlying health conditions, with one study demonstrating worsened outcomes among diabetic patients. ${ }^{[8]}$ Prompt administration of intravenous antibiotic therapy plays a key role in the acute management of endogenous endophthalmitis. ${ }^{[7]}$ Several studies are investigating new molecules with bette antibacterial activity and less ocular toxicity Until these results are available, the evaluation and therapeutic choice remain the surgeon's best judgment. 
In our study, we found 9 cases of endophthalmitis in the total acute medical care department (around 6,000 patients in 3 years). We found a higher incidence in advanced age group (10 cases out of 14 in the 'more than 40 years old' age group), bilateral involvement in 2 patients (out of 14) - $13 \%$. We did not find much difference between incidences in the right and left eye.

The majority of patients have associated predisposing conditions. Several studies have shown diabetes mellitus to be the most common association. (In one series, it was in the range $80-90 \%)$. We also found endophthalmitis in $6(42 \%)$ diabetic patients out of 14. In different published series, fungal organisms account for more than half of the cases of endogenous endophthalmitis in which candida albicans is the most frequent cause $(75-80 \%)$ and in our study fungal organism was found in 4 cases out of 8 culture-positive cases, in which 3 (75\%) cases were of candida.[5] Debate exists over the intraocular aspiration / biopsy of endogenous endophthalmitis. Blood, urine, cerebrospinal fluid, complete blood count with differential (evaluating for signs of infection, elevated white count), (erythrocyte sedimentation rate (evaluating for rheumatic causes, chronic infections or malignancy), blood urea nitrogen (evaluating for renal failure or patients at increased risk), wound culture and smear may be indicated to locate the original infection and document systemic involvement. In one study, vitreous showed the highest yield (74\%), followed by blood culture (72\%). ${ }^{[9]}$ In our study also, non-ocular culture was positive in 6 cases out of 14 (42\%); however, ocular culture was positive in 8 cases out of $12(66 \%)$.

Role of intravitreal antibiotic injections and vitrectomy has been debated, but recent reports suggested that cases of marked intraocular infection (vitritis preventing visibility of optic nerve head or macula) should be managed similarly to cases of acute postoperative infectious endophthalmitis. In our study, 12 eyes were given intravitreal antibiotics and subsequently all eyes with fungal endophthalmitis (3 eyes) underwent vitrectomy with injection of amphotericin B. In our study, we found that there was a definite improvement in ocular signs and visual acuity after vitrectomy and intra-vitreal injection of antibiotics.

\section{CONCLUSION}

Our experience suggests that endogenous endophthalmitis is generally associated with poor visual outcome. Prompt treatment with intravitreal antibiotics and vitrectomy can result in improvement in ocular signs and visual acuity in majority of the patients.

Patients with culture-positive endogenous endophthalmitis are more likely to have fungal isolates with a predominance of candida. Fungal endopthalmitis may be associated with a poor visual outcome as compared to bacterial endophthalmitis; however, this claim needs to be substantiated with further studies.

\section{REFERENCES}

1. Okada AA, Johnson RP, Liles WC, D'Amico DJ, Baker AS. Endogenous bacterial endophthalmitis.
Report of a ten-year retrospective study. Ophthalmology 1994;101:832-8.

2. Anand AR, Therese KL, Madhavan HN. Spectrum of aetiological agents of postoperative endophthalmitis and antibiotic susceptibility of bacterial isolates. Indian $\mathrm{J}$ Ophthalmol 2000;48:123-8.

3. Das T, Kunimoto DY, Sharma S, Jalali S, Majji AB, Nagaraja Rao T, et al. Relationship between clinical presentation and visual outcome in postoperative and posttraumatic endophthalmitis in south centra India. Indian J Ophthalmol 2005;53:5-16.

4. Greenwald MJ, Wohl LG, Sell CH. Metastatic bacterial endophthalmitis: A contemporary reappraisal. Surv Ophthalmol 1986;31:81-101.

5. Schiedler V, Scott IU, Flynn HW Jr, Davis JL, Benz MS, Miller D. Culture-proven endogenous endophthalmitis: Clinical features and visual acuity outcomes. Am J Ophthalmol 2004;137:725-31.

6. Barza M, Pavan PR, Doft BH, Wisniewski SR, Wilson LA, Han DP, et al. Evaluation of

microbiological diagnostic techniques in postoperative endophthalmitis in the Endophthalmitis Vitrectomy Study. Arch Ophthalmo 1997;115:1142-50.

7. Results of the Endophthalmitis Vitrectomy Study. A randomized trial of immediate vitrectomy and of intravenous antibiotics for the treatment of postoperative bacterial endophthalmitis. Endophthalmitis Vitrectomy Study Group. Arch Ophthalmol 1995;113:1479-96.

8. Gupta A, Gupta V, Gupta A, Dogra MR, Panda SS, Ray $P$, et al. Spectrum and clinical profile of post cataract surgery endophthalmitis in North India. Indian J Ophthalmol 2003;51:139-45.

9. Donahue SP, Kowalski RP, Jewart BH, Friberg TR Vitreous cultures in suspected endophthalmitis. Biopsy or vitrectomy? Ophthalmology 1993;100:452-5

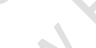

Source of Support: Nil, Conflict of Interest: None declared.

\section{ERRATUM}

Indian Journal of Medical Sciences, October 2006; Vol 60:Issue 10.

Title: Angiotensin converting enzyme inhibitors and aortic arch obstructive malformations Page: 417

Maliheh K, Abdorrazagh K, Armen K, Reza S.

\section{should read as}

Kadivar M, Kiani A, Kocharian A, Shabanian R. 